\title{
Métrologie du tritium dans différentes matrices : cas du tritium organiquement lié (TOL)
}

\author{
N. BAGLAN ${ }^{1}$, E. ANSOBORLO ${ }^{2}$, C. COSSONNET $^{3}$, L. FOUHAL $^{4}$, I. DENIAU $^{5}$, \\ M. MOKILI ${ }^{5}$, A. HENRY ${ }^{6}$, E. FOURRÉ ${ }^{7}$, A. OLIVIER ${ }^{8}$
}

(Manuscrit reçu le 12 décembre 2009, accepté le 22 avril 2010)

RÉSUMÉ La mesure du tritium sous ses différentes formes (gaz (HT), eau (HTO), ou solide (hydrures)), est une étape importante pour l'évaluation des risques sanitaires et environnementaux associés et in fine son estimation dosimétrique. Dans les échantillons du règne végétal ou animal, le tritium est associé à la fraction eau libre et est inclus dans les composés organiques sous forme de tritium organiquement lié (TOL). Dans ce cas, 2 formes existent : (i) la fraction dite échangeable ou labile (TOL-E) liée à des atomes d'oxygène et d'azote ; (ii) la fraction dite non échangeable (TOL-NE) liée aux atomes de carbones. La technique de référence pour l'analyse du tritium est la scintillation liquide permettant de mesurer des concentrations de l'ordre de quelques Bq. $\mathrm{L}^{-1}$. Les normes (AFNOR, ISO) publiées à ce jour ne concernent que l'analyse du tritium dans l'eau. Seule une méthode CETAMA aborde la mesure du TOL dans des milieux biologiques. Cette méthode a été testée depuis 2001 via des circuits d'intercomparaisons sur des échantillons d'herbe prélevés dans l'environnement. Concernant l'analyse du tritium dans les eaux, les principaux points forts sont la fiabilité de cette analyse pour des concentrations de l'ordre de $1 \mathrm{~Bq} . \mathrm{L}^{-1}$, la robustesse et la simplicité de mise en œuvre. Les points faibles sont essentiellement liés à des problèmes de bruit de fond, de conservation et de contamination des échantillons. Concernant l'analyse du TOL, l'analyse est fiable pour des valeurs de l'ordre de $50 \mathrm{~Bq} \cdot \mathrm{kg}^{-1}$ d'échantillon frais. Les points faibles sont des problèmes de contamination, de reproductibilité, de temps d'analyse ( 2 à 6 jours) et l'absence de matériaux de référence. La difficulté à ce jour est la séparation entre TOL-E et TOL-NE qui doit être validée expérimentalement.

ABSTRACT Tritium metrology within different media: focus on organically bound tritium (OBT).

The measurement of tritium in its various forms (mainly gas (HT), water (HTO) or solid (hydrides)), is an important key step for evaluating health and environmental risks and finally, dosimetry assessment. In vegetable or animal samples, tritium is

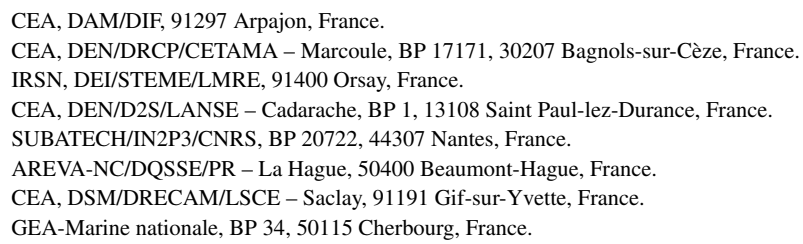


often associated with the free water fraction, but may be included in the organic form as organically bound tritium (OBT). In this case, 2 forms exist: (i) a fraction called exchangeable or labile (E-OBT), bound to oxygen and nitrogen atoms, and (ii) a so-called non-exchangeable fraction (NE-OBT) bound to carbon atoms. The main technique for tritium analysis is liquid scintillation, which enables one to measure concentrations in the range of several Bq. $\mathrm{L}^{-1}$. The standards (AFNOR, ISO) published to date relate only to tritium analysis in water. Only one CETAMA method addresses OBT analysis in biological environments. This method has been tested since 2001 through intercomparison circuits on grass samples collected from the environment. Regarding tritium analysis in water, the strengths are reliability of this analysis at low concentrations (order of $\mathrm{Bq} . \mathrm{L}^{-1}$ ), robustness and simplicity, and weaknesses are linked to problems of background, conservation and contamination of samples. Concerning OBT analysis, the analysis is reliable for values around $50 \mathrm{~Bq} \cdot \mathrm{kg}^{-1}$ of fresh sample. The weaknesses are problems of contamination, reproducibility, analysis time ( 2 to 6 days) and lack of reference materials. The difficulty to date is the separation between E-OBT and NE-OBT, that will need experimental validation.

Keywords: Tritium / tritiated water / organically bound tritium / analysis / environment

\section{Introduction}

Le tritium est un émetteur $\beta$ ayant une énergie moyenne de 5,68 $\pm 0,01 \mathrm{keV}$, une période de 12,312 $\pm 0,025$ ans (LNE-LNHB/CEA, 2006) et une activité spécifique de $3,58 \times 10^{14}$ Bq. ${ }^{-1}$.

Sa présence dans l'environnement a deux origines : soit naturelle, suite à l'interaction des neutrons cosmiques sur l'azote ou l'oxygène, soit anthropique, avec plusieurs sources possibles comme l'émission et la décroissance des retombées liées aux anciens essais nucléaires atmosphériques, les installations de traitement et recyclage de combustibles irradiés, les réacteurs nucléaires et quelques sources industrielles (Belot et al., 1996 ; Guétat et al., 2008; LebaronJacobs et al., 2009).

Il peut exister sous des formes physiques très différentes: sous forme d'hydrogène gaz (HT), d'eau ou de vapeur d'eau (HTO) ou de méthane $\left(\mathrm{CH}_{3} \mathrm{~T}\right)$. Dans les échantillons du règne végétal ou animal, on le trouve souvent associé à la fraction eau libre, mais il peut être inclus dans les composés organiques sous forme de tritium organiquement lié (TOL). Dans ce cas, 2 formes existent : (i) la fraction dite échangeable ou labile (TOL-E) où il est lié à des atomes d'oxygène, de souffre et d'azote ; (ii) la fraction dite non échangeable (TOL-NE) où il est lié aux atomes de carbone (Belot et al., 1996). Enfin dans certains cas (ITER) il peut être présent sous forme solide d'hydrures métalliques ( $\mathrm{Zr}, \mathrm{Ti}, \mathrm{Be}, \mathrm{Hf}, \mathrm{C} . .$. ) (Maubert et Di Pace, 2008). 
L'eau est intégrée à la matière organique des plantes lors de la photosynthèse, suivie de nombreuses réactions biochimiques qui conduisent à un peu plus d'un million de composés organiques. Chaque molécule a un devenir particulier, très différent de l'eau de constitution des cellules.

La commission internationale de protection radiologique (ICRP, 1979) considère par simplification que le tritium organique est pour moitié retransformé rapidement en eau $\left(T_{1 / 2}=10 \mathrm{j}\right)$ et pour moitié, a une durée de vie égale à la durée de vie moyenne observée pour le carbone $\left(T_{1 / 2}=40 \mathrm{j}\right)$. De ce fait l'exposition par unité de tritium incorporée est plus forte pour le tritium organiquement lié que pour le tritium de l'eau (facteur 2 à 3 selon l'âge). Il convient alors de distinguer les deux formes chimiques et d'être précis dans l'utilisation du « concept » de tritium organiquement lié. Il s'agit d'un ensemble de molécules de la ration alimentaire.

La mesure du tritium sous ses différentes formes est donc une étape importante pour l'évaluation des risques sanitaires et environnementaux et in fine pour l'estimation dosimétrique.

La Commission d'établissement des méthodes d'analyse (CETAMA) au sein du CEA, a pour mission de contribuer à la qualité des résultats de mesures et analyses en menant différentes actions appropriées, dont l'organisation et l'interprétation de circuits d'intercomparaison. Le but est d'accompagner les démarches d'amélioration et de validation des méthodes analytiques. Ainsi la demande des laboratoires chargés de la surveillance dans l'environnement a conduit à la création d'un groupe de travail (GT 31 «tritium») sur l'analyse du tritium dans l'environnement (tritium libre et TOL), et à l'organisation de circuits d'intercomparaison sur échantillons frais ou secs prélevés dans l'environnement.

\section{Intérêts et besoins métrologiques de la quantification du tritium organiquement lié (TOL)}

Le séminaire « Tritium » organisé par la SFRP en septembre 2009 et réunissant plus de 200 personnes concernées par cette thématique (exploitants, radioprotectionnistes, chercheurs, métrologistes, représentants de l'ASN, et des CLI...), a suscité de nombreux questionnements sur l'étiologie du tritium (Métivier et al., 2009). Parmi ces questions, les problèmes, liés à la bio-distribution et la bioaccumulation du tritium et donc à la spéciation de ce radionucléide chez l'homme et dans l'environnement, ont souligné un besoin en analyse dans le domaine des faibles valeurs (quelques Bq. $\mathrm{L}^{-1}$ ) et plus précisément en ce qui concerne le tritium organiquement lié (TOL). 


\section{Les méthodes d'analyse du tritium libre et du TOL : état de l'art}

\subsection{Aspect normatif}

Les normes rattachées à la thématique environnementale sont peu nombreuses et deux d'entre elles se rapportent aux rejets (AFNOR, 1999, 2005a). Les autres normes répertoriées sur le site de l'AFNOR (Association française de normalisation) traitent de l'analyse du tritium dans les eaux. Signalons la norme internationale PR NF ISO 9698 « Water quality - Determination of tritium activity concentration - liquid scintillation counting method » (ISO, 1989, 2009) en cours de validation (2009-2010) au niveau français, ainsi que la norme NF M60-802 «Mesure de la radioactivité dans l'Environnement-Eau» de juillet 2000, qui comporte 2 parties relatives au tritium :

- partie 1 : mesurage de l'activité des émetteurs béta dans les eaux par scintillation liquide cas particulier du tritium (AFNOR, 2000);

- partie 3 : mesurage de l'activité des émetteurs béta dans les eaux par scintillation liquide cas particulier de la présence simultanée du tritium et du carbone-14 (AFNOR, 2002).

Ces deux normes ne traitent pas de l'analyse du tritium organiquement lié (TOL). Par contre, la CETAMA a élaboré et testé une méthode d'analyse (CETAMA 384, 2001) intitulée « Dosage du tritium dans des matrices biologiques », au sein d'un groupe de travail (GT 31) «Analyse de radionucléides dans l'environnement : sous-groupe Tritium ».

\subsection{Prélèvement et traitement des échantillons}

Les échantillons végétaux prélevés et utilisés, par exemple dans le cadre de circuits intercomparaison, sont en général des herbes et/ou des feuilles d'arbres d'une même espèce abondante dans la zone échantillonnée. À l'issue du prélèvement, les échantillons sont généralement séchés afin d'en éliminer l'eau libre. Si les échantillons n'ont pas pu être séchés, le stockage dans des emballages étanches ne doit pas être trop long, au plus quelques jours, afin d'éviter une dégradation de la matière organique qui en modifierait considérablement la structure. Le transport doit évidemment éviter le passage par un centre utilisant ou rejetant du tritium. Lorsque cela est possible, l'échantillon est traité et analysé directement.

Les différentes formes du tritium qui coexistent dans les échantillons environnementaux doivent être séparées (Fig. 1). La préparation des échantillons dépend de la mesure souhaitée. La mesure du tritium total est effectuée par 


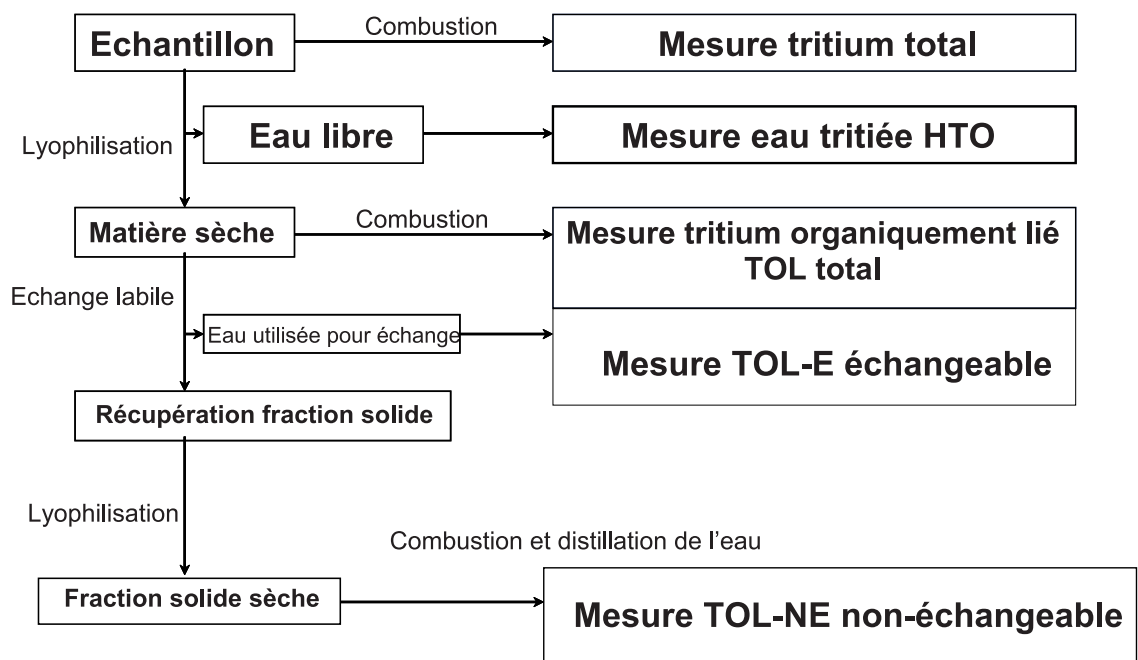

Figure 1 - Schéma d'analyse du tritium dans les échantillons de l'environnement.

Tritium analysis diagram in environmental samples.

combustion directe de l'échantillon. La séparation des différentes fractions nécessite ensuite des étapes plus ou moins nombreuses et complexes.

\subsubsection{Extraction de l'eau libre et mesure du tritium sous sa forme HTO}

La lyophilisation (ou cryodessiccation sous vide) est la méthode actuellement la plus utilisée par les laboratoires pour éliminer l'eau libre et a généralement remplacé la distillation azéotropique qui utilisait des solvants organiques. Elle consiste à congeler rapidement l'échantillon et à le placer dans une enceinte où règne un vide poussé ( $<1$ mbar). L'eau de l'échantillon se dégage alors par sublimation et est récupérée dans un piège froid. La lyophilisation peut être réalisée au laboratoire ou bien sur le terrain à l'aide d'un dispositif mobile. La durée de l'extraction est liée à la masse d'eau minimale nécessaire au comptage par scintillation liquide $(10 \mathrm{~g})$, d'où une durée moyenne de $24 \mathrm{~h}$.

Une fois l'échantillon sec, il est possible de réaliser une combustion pour mesurer le tritium organique total ou bien de continuer le traitement afin de différencier le tritium organique lié échangeable et le tritium organique lié non échangeable. 


\subsubsection{Extraction du tritium organiquement lié échangeable (TOL-E)}

Dans les végétaux, le tritium organiquement lié échangeable (TOL-E) est très labile et s'échange rapidement avec l'hydrogène de la vapeur d'eau atmosphérique ou de l'eau liquide (Belot et al, 1996). Cette propriété est utilisée pour éliminer le tritium par échange isotopique avec de l'hydrogène « léger ». Lors de cette opération appelée « échange labile », l'échantillon préalablement séché et broyé est plongé dans de l'eau sans tritium. Un équilibre isotopique s'établit entre le tritium organique labile et l'eau d'échange, rajoutée en excès (facteur 10 environ) jusqu'à ce que l'activité résiduelle en tritium organique échangeable soit devenue négligeable. Suite à cette opération, l'eau d'échange est éliminée par filtration ou bien par centrifugation pour des échantillons préalablement réduit en poudre. Les échantillons étant humides à la fin de cette étape, un séchage par lyophilisation est ensuite nécessaire. À l'issue de cette étape, l'échantillon ne contient désormais plus que la fraction non échangeable du tritium organiquement lié et peut être calciné dans un four tubulaire si la mesure est réalisée par scintillation liquide ou introduit dans un ballon de quartz dans l'optique d'une mesure par spectrométrie de masse.

\subsubsection{Extraction du tritium organiquement lié non échangeable (TOL-NE)}

Lorsque l'eau libre et le tritium organiquement lié échangeable ont été extraits, il reste encore du tritium sous forme de TOL-NE. Cette fraction est extraite par combustion dans un four tubulaire de diamètre suffisant pour le traitement d'échantillons de masse élevée (jusqu'à $40 \mathrm{~g}$ ) afin de récupérer la masse d'eau de combustion nécessaire à une mesure optimale par scintillation liquide (Pointurier et al., 2004 ; Baglan et al., 2005). Une photo du four utilisé est présentée en figure 2.

Le four est composé d'un tube en quartz inséré dans deux unités chauffantes (Fig. 3). Le tube en quartz est balayé par un flux constant d'oxygène pur et sec. L'échantillon, mis dans la nacelle de quartz, est placé dans le tube au niveau de la première unité chauffante $\left(450{ }^{\circ} \mathrm{C}\right)$.

Le tube contient aussi, au niveau de la deuxième unité chauffante $\left(850{ }^{\circ} \mathrm{C}\right)$, un mélange catalyseur à base d'oxyde de cobalt. La première unité chauffante est mobile de façon à ce que la combustion se déroule à une allure optimale. La combustion ne doit pas être menée trop rapidement afin de limiter le risque de déflagration et de laisser suffisamment de temps pour l'oxydation catalytique des gaz de combustion. En revanche, une combustion menée trop lentement peut conduire à une combustion fractionnée par extinctions de flammes. La durée de combustion optimale est dépendante de la nature de l'échantillon mais de manière générale elle ne dépasse pas une heure pour les végétaux mais peut atteindre plusieurs heures pour des échantillons plus gras comme les poissons, la viande. 


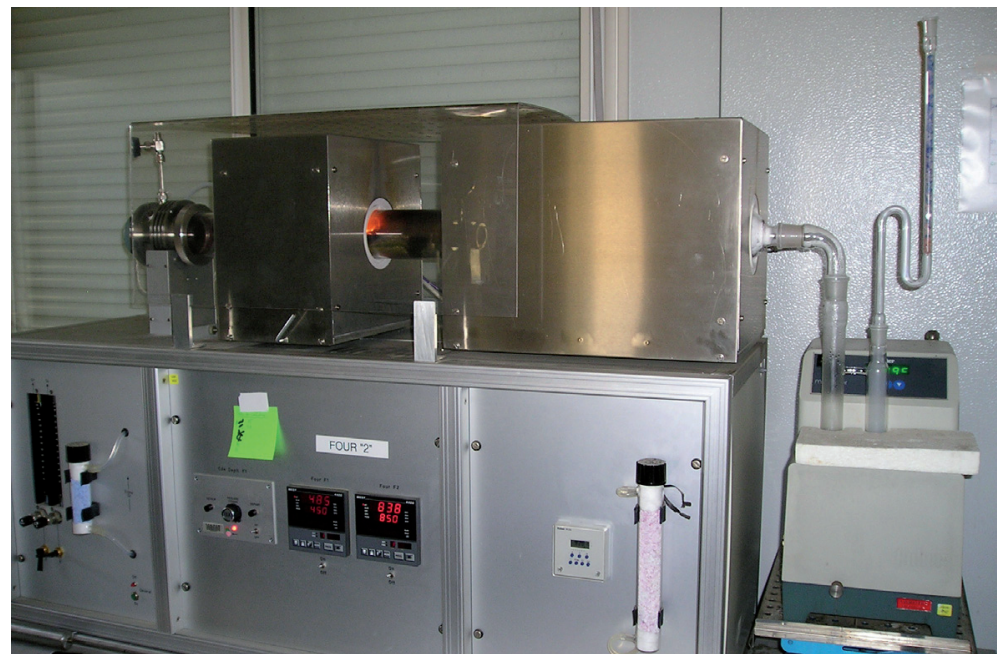

Figure 2 - Four à combustion utilisé en laboratoire pour l'analyse du tritium.

Combustion oven currently used in laboratories for tritium analysis.

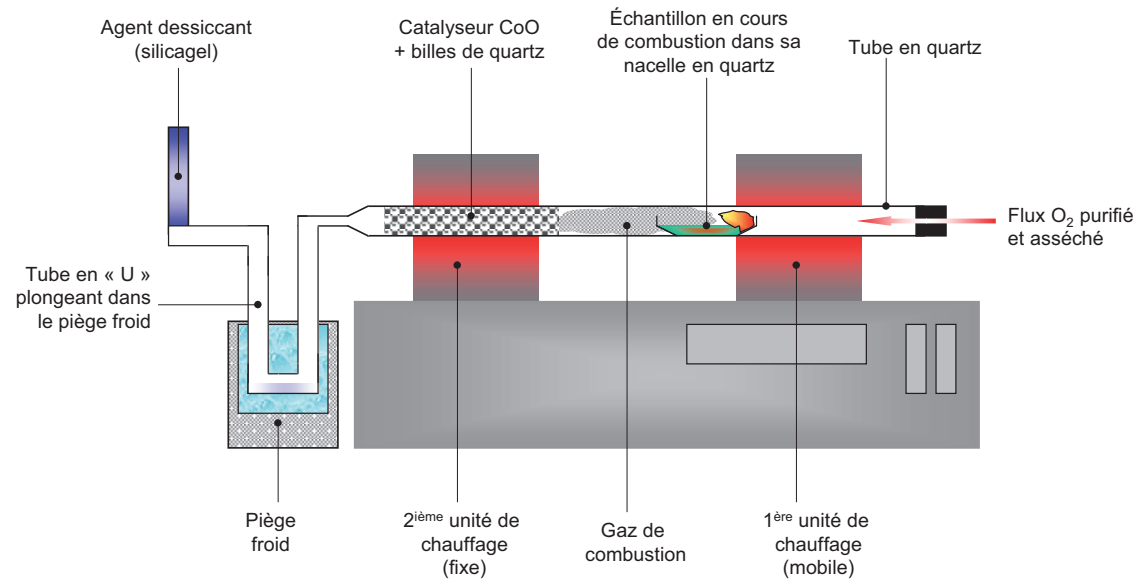

Figure 3 - Schéma de principe de la combustion des échantillons tritiés.

Principle diagram for tritium sample combustion.

La première unité permet donc une combustion complète et lente de l'échantillon qui est entièrement réduit en cendres. La deuxième unité chauffante permet l'oxydation catalytique des gaz de combustion pour former l'eau de combustion recueillie dans un piège froid placé à la sortie du tube. Le piège froid 
est constitué d'un « tube en $\mathrm{U}$ » plongeant dans une solution refroidie à $-20{ }^{\circ} \mathrm{C}$ environ. L'eau récupérée est généralement acide $(\mathrm{pH} \approx 1)$. Pour réaliser la mesure par scintillation liquide, il est indispensable de la neutraliser (addition de peroxyde de sodium $-\mathrm{Na}_{2} \mathrm{O}_{2}$ ) avant distillation. La distillation permet ensuite d'éliminer les divers sels formés lors de la combustion avant l'ajout du liquide scintillant. Le mélange une fois homogénéisé est introduit dans le détecteur pour comptage et détermination de la concentration en tritium (Pointurier et al., 2003).

\section{Techniques d'analyse}

La technique de référence utilisée couramment en routine pour l'analyse du tritium est la scintillation liquide (Theodorsson, 1999 ; Cassette, 2004 ; Pointurier et al., 2004) permettant de mesurer des concentrations de l'ordre de quelques Bq.L ${ }^{-1}$ pour les compteurs bas bruit de fond.

Son principe de base est l'utilisation d'un mélange scintillant qui permet de transformer le rayonnement $\beta$ (e.g. tritium) en photons, qui sont collectés sur un photomultiplicateur et mesurés hors du liquide. Cette technique repose sur une cascade de transfert d'énergie dont les vecteurs sont le rayonnement ionisant, les électrons, les photons lumineux et les électrons du photomultiplicateur. Les appareils modernes de comptage par scintillation liquide permettre d'atteindre des limites de détection relativement faibles autorisant la mesure d'activités atteignant de l'ordre de 1 Bq.L ${ }^{-1}$ (Pointurier et al., 2003) Les inconvénients principaux de cette technique résident dans son rendement énergétique global qui est faible et variable en fonction de la composition de la source scintillante. Cela impose de calculer le rendement de détection pour chaque condition de mesure.

La spectrométrie de masse (Clarke et al., 1976 ; Jean-Baptiste et al, 1992 ; Surano et al., 1992), utilisant la décroissance du ${ }^{3} \mathrm{H}_{\mathrm{en}}{ }^{3} \mathrm{He}^{+}$, suivie d'une détection par un multiplicateur d'électrons fonctionnant en mode comptage, est une technique complémentaire, permettant d'atteindre des valeurs beaucoup plus faibles $\left(<0,5 \mathrm{~Bq} . \mathrm{L}^{-1}\right)$, mais avec des temps de décroissance avant comptage beaucoup plus longs (quelques mois).

\subsection{Calcul des activités en tritium et formules utilisées en scintillation liquide}

Indépendamment de la fraction analysée, l'activité déterminée est dans un premier temps l'activité massique du tritium dans l'eau. 
L'activité massique $A_{m}^{H T O}$ d'un échantillon en $\mathrm{Bq} \cdot \mathrm{kg}^{-1}$ est calculée à l'aide de la formule (1) :

$$
A_{m}^{H T O}=\frac{10^{3}}{60} \frac{\left(n-n_{0}\right)}{\varepsilon m}
$$

l'incertitude finale $A_{m}^{H T O}$ (2) sur le résultat de cette formule (1) est donnée avec un facteur d'élargissement k égal à 2

$$
U_{A_{m}^{H T O}}=2 u_{A_{m}^{H T O}}=2 \sqrt{\frac{\frac{n}{t}+\frac{n_{0}}{t_{0}}}{\left(n-n_{0}\right)^{2}}+\left(\frac{u_{\varepsilon}}{\varepsilon}\right)^{2}+\left(\frac{u_{m}}{m}\right)^{2}}
$$

avec : $m$, masse d'eau $(\mathrm{g}) ; n$ et $n_{0}$, taux de comptage de l'échantillon et du blanc (coups par minute ou cpm) ; $t$ et $t_{0}$, temps de comptage de l'échantillon et du blanc (min) ; $\varepsilon$, rendement de détection, nombre sans dimension compris entre 0 et $1 ; u_{\varepsilon}$, incertitude sur le rendement; $u_{m}$, incertitude sur la masse; $10^{3}$, facteur de conversion $\left(\mathrm{g}^{-1}\right.$ en $\left.\mathrm{kg}^{-1}\right), 60$, facteur de conversion (min en $\mathrm{s}$ ).

Lors de l'analyse du tritium présent dans l'eau, cette unique formule suffit. Par contre, pour des échantillons environnementaux l'activité mesurée dans l'eau (de lyophilisation ou de combustion) est ramenée à celle présente dans l'échantillon frais (ou sec). Les formules présentées ci-après, à titre d'illustration, pour le TOL-NE s'appliquent donc également pour le TOL.

\subsection{Calcul de l'activité en tritium organiquement lié non échangeable}

L'activité massique peut aussi être exprimée en $\mathrm{Bq}_{\mathrm{kg}}{ }^{-1}$ de matière sèche ou de matière fraîche. Il faut alors relier la mesure de l'activité dans l'eau de combustion à l'activité de la matière sèche ou fraîche par l'intermédiaire du rendement hydrogène pendant la combustion.

L'activité $A_{s}^{T O L-N E}$ de la fraction tritium organiquement lié non échangeable en Bq. $\mathrm{kg}^{-1}$ de matière sèche s'exprime comme suit :

$$
A_{s}^{T O L-N E}=A_{m}^{H T O} \frac{r_{H}^{e c h}}{r_{H}^{e a u}}
$$

et l'incertitude $U_{A_{s}^{T O L-N E}}$ associée par la relation :

$$
U_{A_{s}^{T O L-N E}}=2 A_{s}^{T O L-N E} \sqrt{\left(\frac{u_{A_{m}^{H T O}}^{H T}}{A_{m}^{H T O}}\right)^{2}+\left(\frac{u_{H}^{\text {eau }}}{r_{H}^{e a u}}\right)^{2}+\left(\frac{u_{H}^{\text {ech }}}{r_{H}^{e c h}}\right)^{2}}
$$

avec : $r_{H}^{e a u}$, pourcentage massique d'hydrogène dans l'eau $; r_{H}^{e c h}$, pourcentage massique d'hydrogène dans la matière organique par échantillon. 
L'activité $A_{f}^{T O L-N E}$ de la fraction tritium organiquement lié non échangeable en Bq. $\mathrm{kg}^{-1}$ de matière fraiche s'exprime comme suit :

et l'incertitude $U_{A_{f}^{T O L-N E}}$ associée par la relation :

$$
A_{f}^{T O L-N E}=A_{m}^{H T O} \frac{r_{H}^{e c h}}{r_{H}^{e a u}} \frac{m_{s}}{m_{f}}=A_{s}^{T O L-N E} \frac{m_{s}}{m_{f}}
$$

$$
U_{A_{f}^{T O L-N E}}=2 A_{f}^{T O L-N E} \sqrt{\left(\frac{u_{A_{s}^{T O L-N E}}}{A_{s}^{T O L-N E}}\right)^{2}+\left(\frac{u_{m_{f}}}{m_{f}}\right)^{2}+\left(\frac{u_{m_{s}}}{m_{s}}\right)^{2}}
$$

avec : $m_{s}$, masse de matière sèche totale récupérée avant la combustion $(\mathrm{g}) ; m_{f}$, masse de matière fraîche introduite au début du traitement $(\mathrm{g})$.

\subsection{Calcul des teneurs en tritium par spectrométrie de masse}

Pour une mesure par spectrométrie de masse, l'échantillon est placé dans un ballon en verre, préalablement purgé, puis scellé (Jean-Baptiste et al., 2010). Le ballon est ensuite stocké le temps nécessaire à l'obtention d'une quantité d'hélium-3 $\left({ }^{3} \mathrm{He}\right)$, par décroissance du tritium $\left(\mathrm{T}_{1 / 2}=12,312 \mathrm{ans}\right)$, suffisante à la mesure.

Les ions ${ }^{3} \mathrm{He}^{+}$sont détectés par un multiplicateur d'électrons fonctionnant en mode comptage. La ligne de base générée principalement par ${ }^{3} \mathrm{He}\left(10^{-19} \mathrm{~mol}\right)$ résiduel dissous dans l'échantillon après pompage est déduite de la mesure de ${ }^{4} \mathrm{He}$ en appliquant le rapport isotopique de l'hélium atmosphérique $1,38 \times 10^{-6}$. Le rapport $T / H$ pour l'échantillon est déduit de la mesure de ${ }^{3} \mathrm{He}$ issu de la décroissance du tritium d'après :

$$
\frac{T}{H}=\frac{{ }^{3} \mathrm{He}}{m[\mathrm{H}]\left(1-e^{-\lambda t}\right)}
$$

avec : $m$, la masse de l'échantillon (en $\mathrm{kg}$ ), $[\mathrm{H}]$, la concentration en hydrogène (en mol. $\mathrm{kg}^{-1}$ ), $\lambda$ la constante radioactive du tritium et $t$ le temps de stockage.

Cette technique permet d'obtenir des limites de détection très faibles $\left(<0,5 \mathrm{~Bq} . \mathrm{L}^{-1}\right)$, intéressantes pour des applications environnementales. Néanmoins, elle est inversement proportionnelle avec des temps de stockage avant mesure généralement supérieures à une journée, incompatibles avec les exigences de la surveillance réglementaire.

\section{Contraintes de l'analyse à bas niveau}

Les critères pour réaliser une analyse tritium de qualité sont nombreux. Parmi ceux-ci, la maîtrise de la mesure et des différents matériels et équipements 
comme : (i) le flaconnage, (ii) une eau de référence, (iii) un étalon d'eau tritiée, (iv) l'étalonnage ou ajouts dosés et (v) la statistique de comptage; sont nécessaires et indispensables à la mise en place d'une procédure analytique validée permettant l'analyse du tritium à bas niveau (quelques Bq. $\mathrm{L}^{-1}$ ).

\subsection{Flaconnage}

Il existe différents types de flacons de comptage qui se distinguent les uns des autres par leur matériau constitutif. Les plus courants sont les flacons de verre et les flacons de polyéthylène. Les flacons de verre permettent d'observer visuellement les solutions mais le bruit de fond engendré par le flacon est plus élevé que celui des flacons de polyéthylène. Cependant, les solvants organiques qui entrent dans la composition chimique des liquides scintillants diffusent à travers le polyéthylène, ce qui accélère considérablement la dégradation du mélange liquide scintillant prise d'essai. Il existe d'autres types de flacons, notamment :

- des flacons de verre pauvres en ${ }^{40} \mathrm{~K}$, de bruit de fond comparable à celui des flacons de polyéthylène ;

- des flacons de polytétrafluoroéthylène (PTFE) ou des flacons de polyéthylène comportant une couche de PTFE sur la surface intérieure du flacon. La diffusion des solvants organiques est moins rapide à travers le PTFE qu'à travers le polyéthylène. Ces flacons peuvent être utilisés pour des comptages longs à très bas niveau (quelques Bq. $\mathrm{L}^{-1}$ ) ;

- des flacons de PTFE-cuivre, dont les parois sont en PTFE et le bouchon en cuivre. Ces flacons présentent le mouvement propre le plus faible de tous, idéal pour les comptages à très bas niveau d'activité. Cependant, ils sont très coûteux, ce qui impose le nettoyage pour réutilisation de ces flacons.

\subsection{Eau de référence}

Une eau de référence est une eau où idéalement l'activité tritium est négligeable devant celle des échantillons à analyser. Dans ce but, il faut privilégier les eaux souterraines non contaminées par les eaux de surface et suffisamment anciennes pour que le tritium naturel (produit dans la haute atmosphère par interaction des neutrons du rayonnement solaire avec de l'azote) ait décru jusqu'à un niveau d'activité négligeable.

Dans le cas de l'eau de référence, les flacons de prélèvements sont des bouteilles en verre de 2 litres et des lacons souples de type cubitainers ${ }^{\circledR}$ en polyéthylène. Les bouteilles en verre et les cubitainers ${ }^{\circledR}$ sont remplis d'argon quelques heures avant le remplissage par de l'eau de référence. Les cubitainers ${ }^{\circledR}$ présentent l'avantage de ne pas laisser rentrer d'air lors des prélèvements d'eau de référence. Des précautions identiques à celles utilisées pour les prélèvements d'échantillons de très faible 
activité en tritium doivent être prises. La date de prélèvement est notée sur chacun des flacons. À l'échelle de plusieurs mois, aucune contamination en tritium des eaux de forage stockées n'a pu être mise en évidence.

L'eau de source des Abatilles (Arcachon, France), est l'eau de référence ( 0,05 Bq. $\left.\mathrm{L}^{-1}\right)$ utilisée par de nombreux laboratoires pour la mesure du tritium dans le cadre de circuits intercomparaison organisés par la CETAMA. D'autres eaux de forage correspondent à des valeurs faibles $\left(\sim 0,2 \mathrm{~Bq} . \mathrm{L}^{-1}\right)$. Les eaux de références sont analysées à l'issue d'une mesure par enrichissement électrolytique* (Theodorsson, 1999), suivie d'une analyse par scintillation liquide ou par spectrométrie de masse.

\section{*L'enrichissement électrolytique (Plastino et al., 2007)}

Son principe est basé sur le phénomène suivant : dans certaines conditions et avec certains types d'électrodes, l'électrolyse d'une solution d'eau tritiée conduit à un dégagement de l'hydrogène tritié plus lent que celui de l'hydrogène. Il existe une grande variété de milieux électrolytiques, de matériaux constitutifs pour les électrodes, de géométries (dimensions du banc d'enrichissement électrolytique, nombre de cellules d'enrichissement comprenant un récipient étanche, une anode et une cathode). Cependant, les principes suivants doivent être respectés :

- les volumes de départ doivent être importants, de l'ordre de quelques centaines de $\mathrm{ml}$ (en général de $250 \mathrm{~mL}$ à $500 \mathrm{~mL}$ ). Le processus d'enrichissement conduit en effet à une forte diminution du volume de la solution et il est nécessaire de conserver en final un volume suffisant pour la mesure par scintillation liquide (soit quelques $\mathrm{mL}$ ) ;

- la solution résiduelle est impossible à mesurer directement par scintillation liquide, car la concentration en électrolyte augmente de façon inversement proportionnelle à la réduction du volume. II est nécessaire de procéder à une purification de cette solution, par neutralisation et distillation de la solution ;

- le processus dure en général plusieurs jours, car la densité d'énergie fournie à chaque cellule doit être suffisamment faible pour empêcher l'ébullition et l'évaporation de la solution. Les cellules doivent ainsi être continuellement refroidies. Elles sont généralement placées dans une enceinte réfrigérée. L'énergie est fournie par des alimentations stabilisées ;

- le processus d'enrichissement génère un mélange de gaz détonants $\left(\mathrm{H}_{2} \mathrm{O} \rightarrow\right.$ $1 / 2 \mathrm{O}_{2}+\mathrm{H}_{2}$ ). Le banc d'enrichissement doit être ainsi muni des sécurités adéquates : système d'évacuation haute des gaz détonants, détecteurs d'explosivité, ...

L'enrichissement électrolytique associé à la scintillation liquide permet en moyenne d'abaisser la limite de détection d'un facteur 10 à 15 par rapport à la scintillation liquide utilisée seule. Grâce au double enrichissement, obtenu en réunissant toutes les solutions enrichies à l'issue d'un $1^{\mathrm{er}}$ enrichissement, dans une même cellule d'électrolyse pour un ultime enrichissement permettant de gagner un facteur 10 à 15 supplémentaire, il est alors possible d'atteindre des limites de détection voisines de $0,01 \mathrm{~Bq} \cdot \mathrm{L}^{-1}$. 


\section{3. Étalon d'eau tritiée}

Une source certifiée d'eau tritiée, doit être utilisée pour la préparation de la série d'étalons de travail destinés à l'établissement de la courbe d'affaiblissement lumineux. Il est en général nécessaire de diluer la source certifiée pour préparer la série d'étalons de travail à affaiblissements lumineux variables.

À noter que l'activité de la source est fournie à une date définie. Il faut donc tenir compte de la décroissance radioactive à chaque utilisation de la source certifiée.

Soient $A(t)$ l'activité tritium en Bq de la source à l'instant $t, A\left(t_{0}\right)$ l'activité tritium en $\mathrm{Bq}$ de la source à l'instant $t_{0}$ et $\mathrm{T}_{1 / 2}$ la période de demi-vie du tritium (4 $497 \pm 9$ jours), l'activité $A(t)$ vaut :

$$
A(t)=A\left(t_{0}\right) \mathrm{e}^{-\ln (2) \frac{\left(t-t_{0}\right)}{\mathrm{T}_{1 / 2}}} .
$$

Les solutions filles utilisées ultérieurement pour l'étalonnage des compteurs sont réalisées par dilution avec l'eau de référence employée pour déterminer le mouvement propre de l'appareil.

\section{4. Étalonnage}

La réalisation de la série d'étalons de travail à affaiblissements lumineux variables doit respecter un certain nombre de similitudes avec les échantillons mesurés :

- même quantité (masse ou volume) de mélange scintillant,

- même rapport entre la prise d'essai et le liquide scintillant,

- même type de liquide scintillant,

- valeur du paramètre d'affaiblissement lumineux des échantillons incluse dans la gamme d'étalonnage.

Une solution alternative à l'utilisation d'une courbe d'affaiblissement lumineux est la réalisation d'ajouts dosés. Alors à chaque échantillon est associé au minimum un échantillon surchargé avec une activité de tritium connue afin de déterminer la quantité de tritium présente dans l'échantillon.

\section{Durée de l'analyse}

L'analyse de la concentration en tritium dans les échantillons de l'environnement nécessite préalablement à la mesure de réaliser un nombre d'étapes plus ou moins grand en fonction de l'information recherchée. Cela se traduit par des phases préanalytiques de durées croissantes : de l'ordre de la journée pour le tritium total ou la fraction HTO mais augmentant jusqu'à trois jours pour le TOL et 6 jours pour 
le TOL-NE. Sachant qu'à chaque combustion correspond un lavage, qu'un banc de distillation comporte environ 5 postes, qu'un blanc de distillation est associé à chaque série de distillation et que les activités à mesurer sont environnementales, le temps d'analyse pour 2 échantillons sera au minimum de 5 jours.

De plus, pour les applications environnementales, le signal obtenu lors de la mesure de l'activité des échantillons est souvent indiscernable de celui du bruit de fond. On introduit alors les notions de seuil de décision (SD) qui donne la probabilité qu'un radionucléide soit détecté alors qu'il est absent de l'échantillon et de limite de détection (LD) qui donne la probabilité qu'un radionucléide ne soit pas détecté alors qu'il est présent dans l'échantillon.

\section{Seuils de décision}

Depuis la parution de la norme ISO 11929 (AFNOR, 2005b), le mode de calcul du seuil de décision (SD) peut être déterminé par trois relations utilisables en scintillation liquide en fonction du référentiel choisi (Fig. 4) : (i) ISO 11929 (AFNOR, 2005b), (ii) Bayes (Vivier et Aupiais, 2005 ; Weise, 1998) et (iii) NF M60-802-1 (AFNOR, 2000). Ce choix peut poser des problèmes à l'utilisateur :

(i) $S D=\frac{k}{t} \sqrt{2 n_{0} t}$;
(ii) $S D=\frac{k}{t} \sqrt{2\left(1+n_{0} t\right)}$
(iii) $S D=\frac{k}{t}\left(1+\sqrt{1+2 n_{0} t}\right)$

dans les conditions usuelles d'un laboratoire, SD varie de 0,45 à 0,48 cpm lors de l'utilisation d'un compteur Packard (Fig. 4a) et entre 0,060 et 0,065 cpm avec un compteur 1220 Quantulus ${ }^{\mathrm{TM}}$ (Fig. 4b). Le seuil de décision étant par définition déterminé avec une incertitude de $100 \%(k=2)$, il est clair ici que la formule de calcul n'a pas d'impact sur la valeur obtenue. Ainsi, même si les notions de SD et LD sont prépondérantes dans le cadre de l'analyse à bas niveau, les critères pour réaliser une bonne analyse tritium sont plus nombreux :

- prélèvement,

- conservation,

- traitement,

- mesure (étalonnage ou ajouts dosés, statistique de comptage).

À chacune de ces étapes correspondent des contraintes inhérentes à la fois au niveau d'activité à quantifier, à l'outil analytique employé et à l'historique du laboratoire. Leur compréhension et leur maîtrise sont des éléments indispensables 


\section{MÉTROLOGIE DU TRITIUM DANS DIFFÉRENTES MATRICES}

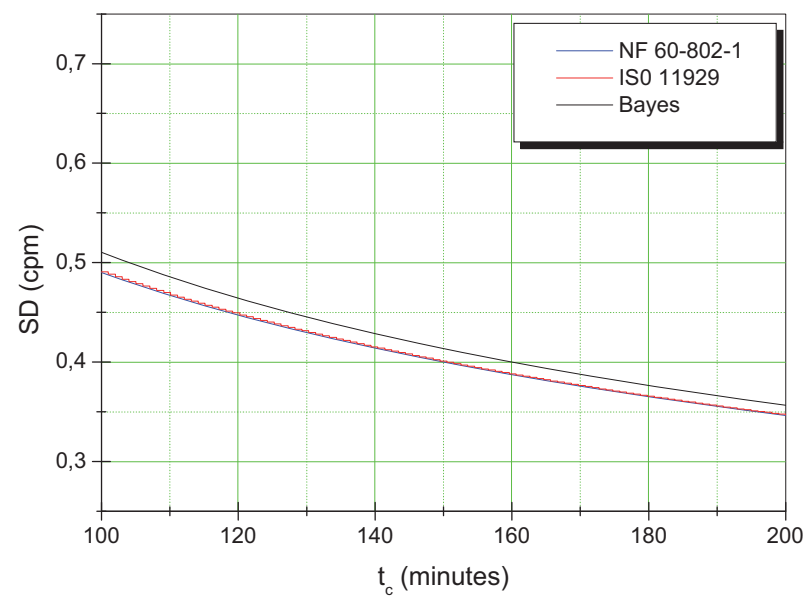

(a)

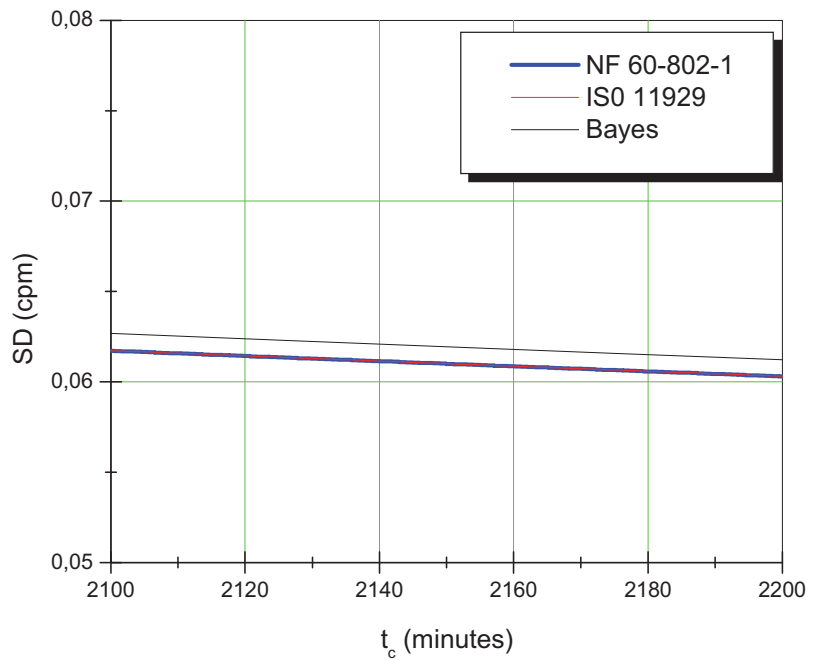

(b)

Figure 4 - Seuil de décision (SD) en cpm $=f(t)$ en bleu SD calculé d'après la formule issue de l'application de la norme 11929, en rouge application de la statistique bayésienne et en noire celle de la norme NF 60-802-1. (a) Compteur Packard $t_{c}=120 \mathrm{~min}, n_{0}=3 \mathrm{cpm}$; (b) compteur Wallac $t_{c}=2160 \mathrm{~min}, n_{0}=1 \mathrm{cpm}$.

Decision threshold $(D T)$ in cpm $=f(t)$. Blue curve: calculated with $N F 11929$ formula; red curve: calculated with bayesian statistic; black curve: calculated with NF-60-802-1 formula. (a) Packard counter $t_{c}=120 \mathrm{~min}, n_{0}=3 \mathrm{cpm}$; (b) Wallac counter $t_{c}=2160 \mathrm{~min}, n_{0}=1 \mathrm{cpm}$. 
à la mise en place d'une procédure analytique validée, permettant l'analyse des niveaux de tritium environnemental.

\section{Exercices d'intercomparaison tritium libre et tritium organique}

Les objectifs des exercices d'intercomparaison sont différents en fonction de l'organisateur. Ils peuvent être élaborés dans un objectif d'obtention d'agrément (Institut de radioprotection et de sureté nucléaire - IRSN) ou en support des analystes afin de valider des méthodes (e.g. la CETAMA ou PROCORAD association pour la promotion des contrôles en radiotoxicologie par exemple).

Dans le premier cas, il s'agit de valider les compétences du laboratoire par un agrément certifiant de la qualité de ses résultats et l'autorisant à réaliser l'analyse en question dans le cadre de la surveillance environnementale. Ainsi l'IRSN organise sur une base biannuelle un circuit de mesure du tritium dans l'eau ( $\left.20 \mathrm{~Bq} . \mathrm{L}^{-1}\right)$, et prévoit en 2010 un premier circuit dans une matrice de lait.

Dans le second cas qui se situe en amont de la normalisation et/ou de la certification, cela permet aux laboratoires participants de tester et valider leur méthode d'analyse sur des plages plus étendues en termes d'activité, de matrice... Il faut noter dans ce cadre, qu' un groupe de travail (GT 31) de la CETAMA organise depuis quelques années des circuits d'intercomparaison de mesure du tritium dans l'eau (10, 50 et $\left.100 \mathrm{~Bq} . \mathrm{L}^{-1}\right)$ et se focalise depuis 2001 sur des intercomparaisons sur l'analyse du tritium organiquement lié (TOL) dans des échantillons d'herbe prélevés dans l'environnement $\left(\sim 50 \mathrm{~Bq} \cdot \mathrm{kg}^{-1}\right)$. L'association PROCORAD (Bérard et al., 1998) organise aussi chaque année des intercomparaisons en radiotoxicologie avec des urines contaminées soit avec de l'eau tritiée (> $\left.500 \mathrm{~Bq} . \mathrm{L}^{-1}\right)$ soit de la thymidine tritiée pour la mesure du TOL (> 10000 Bq.L $\left.{ }^{-1}\right)$.

Citons au niveau international certains organismes comme l'AIEA (Autriche), l'USDOE (USA), l'AECL (Canada) ou encore le NIRS (Japon) qui organisent des circuits d'intercomparaison principalement dans l'eau, mais aussi mesure du TOL dans des matrices environnementales et dans un cas particulier sur des tissus humains (Hisamatsu et al., 1992).

\section{Bilan d'exercices d'intercomparaison pour la mesure du tritium organisés par la CETAMA}

\subsection{Circuit d'intercomparaison eau tritiée (2001-2002)}

Un circuit d'intercomparaison sur des échantillons d'eau tritiée à différentes concentrations $\left(10,50\right.$ et 100 Bq.L ${ }^{-1}$ et la fourniture d'une eau de référence des 
TABLEAU I

Synthèse des résultats du circuit intercomparaison tritium dans l'eau pour 3 échantillons de concentration différente.

Synthesis of tritiated water intercomparison results for 3 different sample concentrations.

\begin{tabular}{|c|c|c|c|}
\hline Échantillons d'eau contenant du tritium & $10 \mathrm{~Bq} \cdot \mathrm{L}^{-1}$ & 50 Bq.L. ${ }^{-1}$ & $100 \mathrm{~Bq} \cdot \mathrm{L}^{-1}$ \\
\hline Moyenne générale & 8,1 & 47,3 & 110,3 \\
\hline Médiane & 8,1 & 46,6 & 110,1 \\
\hline Écart type répétabilité & 0,9 & 2,1 & 3,6 \\
\hline Intervalle de Confiance de répétabilité $(k=2)$ & 1,9 & 4,2 & 7,1 \\
\hline Écart type reproductibilité & 1,2 & 3,4 & 5,9 \\
\hline Intervalle de Confiance de reproductibilité $(k=2)$ & 2,5 & 6,9 & 11,9 \\
\hline \multicolumn{4}{|l|}{ Données caractéristiques du circuit } \\
\hline$p$ essais & 18 & 17 & 18 \\
\hline$N$ résultats & 87 & 82 & 88 \\
\hline$N^{\prime}$ & 82 & 77,1 & 83,1 \\
\hline \multicolumn{4}{|l|}{ Valeurs relatives $(\%)$} \\
\hline Écart type répétabilité & $11 \%$ & $4 \%$ & $3 \%$ \\
\hline Intervalle de confiance de répétabilité $(k=2)$ & $23 \%$ & $9 \%$ & $6 \%$ \\
\hline Écart type reproductibilité & $15 \%$ & $7 \%$ & $5 \%$ \\
\hline Intervalle de confiance de reproductibilité $(k=2)$ & $30 \%$ & $15 \%$ & $11 \%$ \\
\hline
\end{tabular}

Abatilles) a été organisé en 2001-2002 par la CETAMA, avec une participation de 18 laboratoires français. Les résultats obtenus sont présentés dans le tableau I pour les 3 niveaux de concentration et dans les figures $5 \mathrm{~A}$ et $5 \mathrm{~B}$ pour 2 niveaux de concentrations analysés $\left(10\right.$ et $\left.100 \mathrm{~Bq} \cdot \mathrm{L}^{-1}\right)$. Ces résultats sont exprimés sous forme de moyenne générale et médiane, accompagnée des écarts types et intervalles de confiance associés exprimés sous forme de répétabilité (mesures faites par un même opérateur, sur un même instrument, avec la même méthode) et de reproductibilité (conditions de mesure changeantes).

Ces résultats montrent que : (i) pour chaque concentration, les écarts type de répétabilité sont inférieurs à ceux de reproductibilité ; (ii) ces écarts augmentent de façon significative lorsque la concentration diminue ; (iii) pour la concentration la plus faible de $10 \mathrm{~Bq} . \mathrm{L}^{-1}$, l'écart type de répétabilité étant de l'ordre de $11 \%$, ceci définit la limite de quantification de cette méthode (Curie, 1999).

En conclusion de ce circuit, la faible dispersion des résultats (écarts types $<10 \%$ ) sur les 18 laboratoires participants montre que la mesure du tritium est correctement maîtrisée dans ce type de matrice et à ces niveaux d'activité. Les 

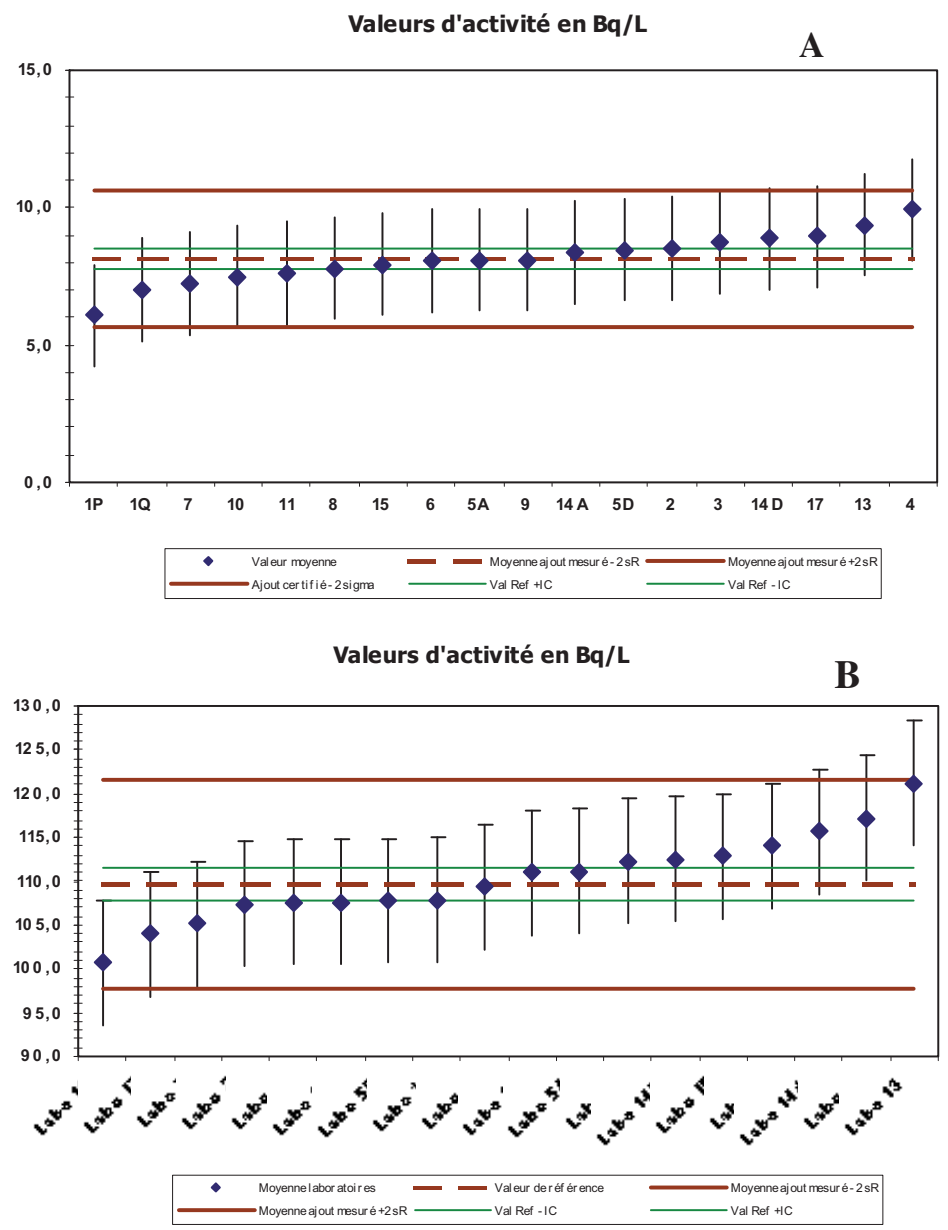

Figure 5 - Résultats d'une intercomparaison (18 participants) d'analyse d'eau tritiée avec 2 concentrations. (A) 8 Bq.L $L^{-1}$, (B) 110 Bq. $L^{-1}$.

Results of a tritiated water analysis intercomparison (18 participants) at 2 concentration levels. (A) 8 Bq. $L^{-1}$, (B) 110 Bq. $L^{-1}$.

caractéristiques du circuit en matière de répétabilité et de reproductibilité indiquent que le niveau de 10 Bq.L ${ }^{-1}$ est relativement proche de la limite de quantification des laboratoires, mais que cette valeur est très dépendante du temps de comptage. 


\subsection{Circuit d'intercomparaison du TOL (2001-2009)}

Afin de valider la méthode CETAMA 384 « Dosage du tritium dans des matrices biologiques : cas du TOL », et de tester certaines étapes décrites dans la figure 1, plusieurs circuits interlaboratoires sur des échantillons biologiques prélevés dans l'environnement ont été organisés entre 2003 et 2009.

Un premier circuit a été organisé en 2001 (6 laboratoires participants) sur un échantillon d'herbe lyophilisé par l'IRSN/LMRE (laboratoire de mesure de la radioactivité dans l'environnement) et sur l'échantillon d'eau de lyophilisation associé. Le but de ce circuit était de tester d'une part la mesure du tritium libre dans l'eau de lyophilisation et d'autre part celle du tritium organiquement lié (TOL) dans l'herbe lyophilisée. Ces résultats ont montré une bonne homogénéité des résultats de mesure du tritium libre mais une forte dispersion des résultats concernant la mesure de TOL.

Un second circuit a donc été organisé en 2004-2006 (5 laboratoires participants) toujours sur un échantillon d'herbe lyophilisé par l'IRSN/LMRE, et plus focalisé sur la mesure du TOL.

Enfin un troisième circuit a été organisé en 2006-2008 (6 à 8 laboratoires participants) de nouveau sur un échantillon d'herbe. Dans ce circuit les laboratoires ont reçu 2 échantillons : l'un correspondant à l'herbe fraîche congelée et l'autre à cette même herbe lyophilisée par le laboratoire Subatech à Nantes. Le but de ce circuit était de tester l'étape de lyophilisation faite par chaque laboratoire sur un échantillon frais par rapport à la même étape réalisée par un seul laboratoire (Subatech).

Les figures 6 et 7 donnent 2 exemples de résultats obtenus d'une part avec les eaux de combustion (Fig. 6) et d'autre part sur la matière sèche (Fig. 7).

Les résultats reportés lors de la dernière intercomparaison de la CETAMA permettent de conclure à un bon accord entre les valeurs obtenues par les différents laboratoires car ces dernières satisfont aux critères de reproductibilité. Ceux-ci soulignent les progrès réalisés par les laboratoires depuis le premier exercice même si l'écart type de reproductibilité $(17$ à $25 \%)$ et donc la limite de reproductibilité sont encore relativement élevés (50 à $78 \%$ ).

Dans ces conditions, il devient difficile de mettre en évidence de manière univoque l'impact potentiel des étapes de traitement de l'échantillon préalables à la mesure. 


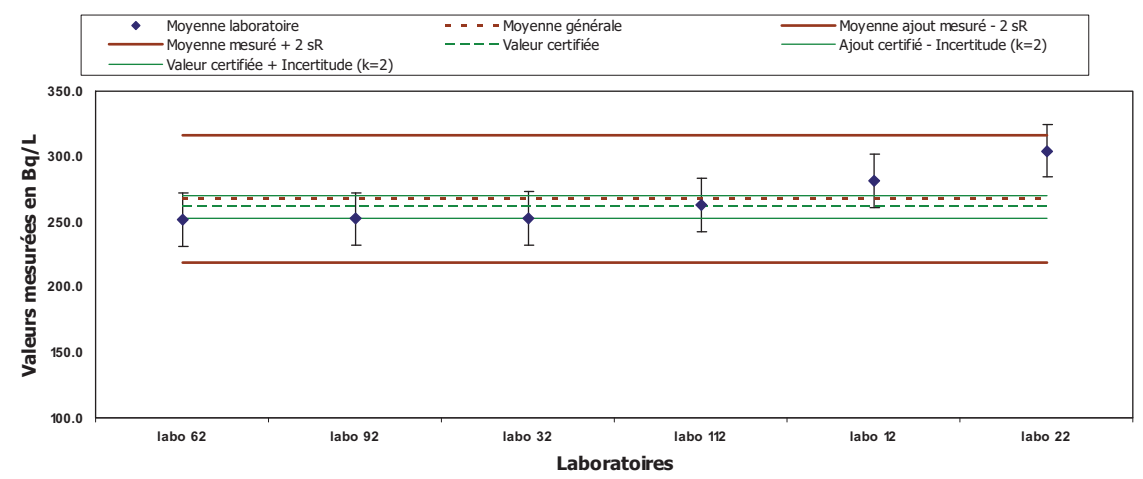

Figure 6 - Résultats d'une intercomparaison (6 participants) d'analyse d'eau de combustion en Bq.L $L^{-1}$, pour tester l'étape de lyophilisation de l'analyse TOL.

Results of a tritium analysis intercomparison (6 participants) on combustion water (Bq. $\left.L^{-1}\right)$ in order to test the lyophilisation step in OBT analysis.

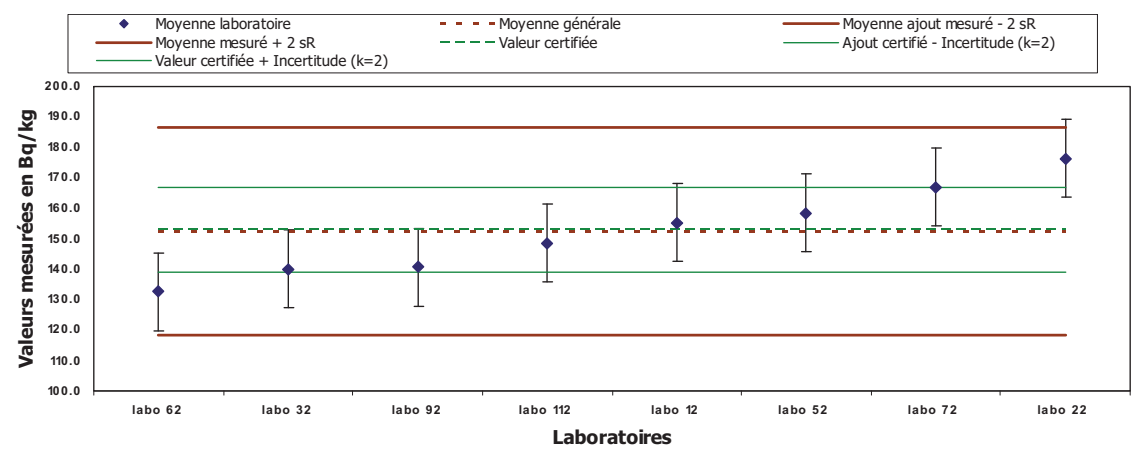

Figure 7 - Résultats d'une intercomparaison (8 participants) d'analyse tritium sur aliquote sèche exprimés en Bq. $\mathrm{kg}^{-1}$ pour tester l'étape de lyophilisation de l'analyse TOL.

Results of a tritium analysis intercomparison (8 participants) on dry matter (Bq.kg-1) in order to test the lyophilisation step in OBT analysis.

Perspectives : une estimation de cet impact nécessite d'améliorer encore nos résultats en terme de dispersion. Dans ce but, un nouvel exercice a été mis en place en 2009. Son objectif principal est de valider une procédure «CETAMA » pour l'analyse du TOL. En parallèle nous essaierons de valider notre procédure analytique par la vérification expérimentale de l'égalité mathématique $T_{\text {total }}=$ HTO + TOL. Après cette étape, il sera alors possible de se focaliser sur l'analyse du TOL-NE et valider une procédure robuste et simple de mise en œuvre à l'horizon 2015. Pour cela, il sera important de bien maîtriser l'étape d'élimination du TOL-E et donc de s'assurer de la non dégradation de la matrice. Dès lors, 
l'égalité TOL $=$ TOL-E + TOL-NE pourra être vérifiée expérimentalement et l'analyse du TOL-NE validée.

\section{Conclusions}

Les normes (AFNOR, ISO) publiées à ce jour ne concernent que l'analyse du tritium dans des échantillons aqueux, et seule une méthode CETAMA aborde la mesure du TOL dans des milieux biologiques. Cette méthode, composée de nombreuses étapes, est testée depuis 2001 via des circuits intercomparaisons sur échantillons d'herbe prélevés dans l'environnement.

Concernant l'analyse du tritium dans les eaux de l'environnement, le point fort est la fiabilité de cette analyse pour des niveaux de concentrations de l'ordre de quelques Bq. $\mathrm{L}^{-1}$, avec possibilité de diminuer la limite de détection soit par enrichissement électrolytique, soit en utilisant la spectrométrie de masse. Les points faibles sont liés à des problèmes de bruit de fond, de conservation et de contamination des échantillons.

Concernant l'analyse du tritium organiquement lié (TOL), l'état des connaissances montre que l'analyse est fiable pour des valeurs de l'ordre de $50 \mathrm{~Bq} \cdot \mathrm{kg}^{-1}$ d'échantillon frais. Les points faibles sont des problèmes de contamination, de reproductibilité liés aux nombreuses étapes analytiques (combustion, lyophilisation...), de temps d'analyse (quelques jours) et l'absence de matériaux de référence. Le point dur à ce jour est la séparation entre TOL-E et TOL-NE qui doit être validée expérimentalement

\section{RÉFÉRENCES}

AFNOR (1999) NF M60-312, Mesure de la radioactivité dans l'environnement-Air - Détermination par scintillation liquide de l'activité volumique du tritium atmosphérique prélevé par la technique de barbotage de l'air dans l'eau.

AFNOR (2000) NF M60-802-1, Mesure de la radioactivité dans l'environnement-Eau. Partie 1: mesurage de l'activité des émetteurs Bêta par scintillation liquide - Cas particulier du tritium.

AFNOR (2002) NF M60-802-3, Mesure de la radioactivité dans l'environnement-Eau. Partie 3 : mesurage de l'activité des émetteurs Bêta par scintillation liquide - Cas particulier de la présence simultanée du tritium et du $14 \mathrm{C}$.

AFNOR (2005a) NF EN60-761-5, Équipements de surveillance en continu de la radioactivité dans les effluents gazeux - Partie 5 : exigences particulières aux moniteurs de tritium.

AFNOR (2005b) NF ISO 11929, Partie 7 : Détermination de la limite de détection et du seuil de décision des mesurages de rayonnements ionisants - Principe fondamentaux et applications.

Baglan N., Alanic G., Pointurier F. (2005) Tritium determination at trace level: which strategy to determine accurately HTO and OBT in environmental samples? Fus. Sci. Techn. 48, 749-754.

Belot Y., Roy M., Métivier H. (1996) Le tritium : de l'environnement à l'homme. EDP Sciences, Les Ulis, 190 p. 
Bérard P., Briot F., Cavadore D., Exmelin L., Fottorino R., Nguyen F. (1998) International radiobioassay quality controls, Radiat. Prot. Dosim. 79, 509-512.

Cassette P. (2004) Mesures de radioactivité par scintillation liquide, P3 analyse et caractérisation, Techniques de l'ingénieur 2552, 1-19.

Clarke W.B., Jenkins W.J., Top Z. (1976) Determination of tritium by mass spectrometric measurement of ${ }^{3} \mathrm{He}$, Int. J. Appl. Rad. Isotopes 27, 515-522.

CETAMA 384 (2001) Dosage du tritium dans des matrices biologiques.

Curie L.A. (1999) Detection and quantification limits: origins and historical overview, Analyt. Chim. Acta 391, 127-134.

Guétat P., Douche C., Hubinois J.C. (2008) Le tritium et l'environnement : sources, mesures et transferts, Radioprotection 43, 547-569.

Hisamatsu S., Ohmura T., Takizawa Y., Katsumata T., Inoe Y., Itoh M., Ueno K., Sakanoue M. (1992) Tritium level in japanese diet and human tissue, J. Radioanal. Nucl. Chem. 156, 89-102.

ICRP Publication 30 (1979) Limits of the intake of Radionucleides by Workers, Part I, Ann. ICRP 2 (3/4).

ISO 9698 (1989) [Revision] Liquid scintillation counting method.

ISO/DIS 9698, ISO/TC147 (2009) Water quality - Determination of tritium activity concentration.

Jean-Baptiste P., Mantisi F., Dapoigny A., Stievenard M. (1992) Design and performance of a mass spectrometric facility for measuring helium isotopes in natural waters and for low level tritium determination by ${ }^{3} \mathrm{He}$ ingrowth method, Appl. Rad. Isotopes 43, 881-891.

Jean-Baptiste P., Fourré E., Dapoigny A., Baumier D., Baglan N., Alanic G. (2010) ${ }^{3}$ He mass spectrometry for very low level measurement of organic tritium in environmental samples, J. Env. Radioact. 101, 185-190.

Lebaron-Jacobs L., Garnier-Laplace J., Lopez B., Adam-Guillermin C., Dublineau I., Roussel-Debet S., Antonelli C., Fievet B., Bailly du Bois P., Masson M. (2009) Toxicologie nucléaire environnementale et humaine, Tritium. Lavoisier, Paris. Chapitre 30, p. 575.

LNE-LNHB/CEA (2006) Table de radionucléides.

Maubert H., di Pace L. (2008) Doses resulting from the inhalation of ITER generated tritiated dust, Radioprotection 43, 13-22.

Métivier H., Hartmann P., Schneider T., Le Guen B., Foulquier L., Michelet M. (2009) Compte rendu des journées tritium organisées par la SFRP. Paris, 23-24 septembre 2009, Radioprotection 44, 519-525.

Plastino W., Chereji I., Cuna S., Kaihola L., de Felice P., Lupsa N., Balas G., Mirel V., Berdea P., Baciu C. (2007) Tritium in water electrolytic enrichment and liquid scintillation counting, Radiat. Meas. 42, 68-73.

Pointurier F., Baglan N., Alanic G., Chiappini R. (2003) Determination of organically bound tritium background level in biological samples from a wide area in the south-west of France, J. Env. Radioact. 68, 171-189.

Pointurier F., Baglan N., Alanic G. (2004) A method for the determination of low level organic bound tritium activities in the environmental samples, Appl. Rad. Isotopes 61, 293-298.

Surano K., Hudson G., Failor R., Sims J., Holland R., MacLean S., Garrison J. (1992) Helium-3 mass spectrometry for lox-level tritium analysis of environmental samples, J. Radioanal. Nucl. Chem. 161, 443-453.

Theodorsson P. (1999) A review of low-level tritium systems and sensitivity requirements, Appl. Rad. Isotopes 50, 311-316.

Vivier A., Aupiais J. (2005) Optimization of the decision threshold for single radioactive counting, Radiochim. Acta 95, 477-492.

Weise K. (1998) Bayesian-statistical decision threshold, detection limit and confidence interval in nuclear radiation measurements, Kerntechnik 63, 214. 